\title{
O Papel do Exercício no Tratamento do Diabetes Melito Tipo 1
}

revisão

\section{ana Claudia R. Ramalho \\ SABRINA SOARES}

Departamento de Clínica Médica da Universidade Federal da Bahia (UFBA), Salvador, BA, Brasil.
Recebido em 29/01/2008 Aceito em 12/02/2008

\section{RESUMO}

Apesar de o exercício ser associado à redução da mortalidade cardiovascular em pacientes com diabetes melito tipo 1 (DM1), vários pontos do tópico exercício em DM1 merecem discussão. Por exemplo: resultados contraditórios têm sido relatados sobre os benefícios da atividade física no controle metabólico desses pacientes. Ainda controverso também é o tipo de exercício mais benéfico neste grupo. Outro ponto refere-se ao melhor ajuste na dose de insulina recomendada para a prática de exercício. Este artigo propõe-se a discutir esses e outros tópicos. O efeito do exercício no controle metabólico em DM1 permanece controverso. Alguns autores encontrando um efeito benéfico na hemoglobina glicada e outros não. Outro ponto controverso é o tipo de exercício mais indicado: resistido ou aeróbico. Existem poucos trabalhos na literatura sobre o efeito do exercício resistido no controle metabólico em DM1. Ainda sem esclarecimento é o efeito do exercício no perfil lipídico em DM1. A intensidade e a duração do exercício, o grau de atividade do indivíduo, a presença de complicações do diabetes, o tempo de doença e o quadro clínico são algumas variáveis que devem ser analisadas antes de se iniciar um programa de exercício. Um esquema de ajuste na dose de insulina e/ou reposição de carboidrato devem ser estratégias utilizadas para se evitar a hipoglicemia relacionada ao exercício. Outros dois aspectos importantes são hidratação e a monitorização que deve ser realizada antes, durante e após o exercício. Ainda neste artigo é discutida avaliação médica pré-exercício. (Arq Bras Endocrinol Metab 2008;52/2:260-267)

Descritores: Diabetes melito tipo 1; Exercício; HbA1c

\section{ABSTRACT}

The Role of Exercise in the Treatment of Type 1 Diabetes.

Although physical activity has been associated with cardiovascular mortality reduction in type-1 diabetes (DM1) patients, many points in the topic 'exercise' deserve a closer look. For example: contradictory data have been reported regarding the benefits of physical activity on metabolic control in these patients. Still contradictory is the type of exercise that brings more benefit in this group. Another issue is the best way of reducing insulin doses for exercise. This article intent to discuss these topics. The effect of exercise on metabolic control in DM1 is still contradictory. Some authors show a beneficial effect on glycated hemoglobin (A1c) and others do not. Another point to be analized is which type of exercise is better for these patients: aerobic or of resistance. There is a lack of information related to the effect of resistance exercises without the aerobic training on metabolic control in type-1 diabetes. The effect of exercise on lipid profile in DM1 is another issue. The intensity and duration of the exercises, the level of physical activity, the duration of 
the disease and the presence of cronic complications are some points that might be taken into consideration before starting an exercise program. Guidelines for the reduction in insulin dose or the use of carbohydrate are strategies to avoid hypoglycemia related to exercises. Hydration and self-monitored blood glucose levels are also very important topics. This article will also discuss the clinical evaluation before doing any exercise. (Arq Bras Endocrinal Metab 2008;52/2: 260-267)

Keywords: Type 1 diabetes; Glycated hemoglobin; Exercise

\section{INTRODUÇÃo}

$\Lambda \mathrm{P}$ PESAR DE O EXERCÍCIO SER associado a redução da mortalidade cardiovascular em pacientes com diabetes melito tipo 1 (DMl) (1), vários pontos do tópico exercício em DMl merecem discussão. Por exemplo: resultados contraditórios têm sido relatados sobre os benefícios da atividade física no controle metabólico desses pacientes. Ainda controverso também é o tipo de exercício mais benéfico neste grupo. Outro ponto referese ao melhor ajuste na dose de insulina recomendada para a prática de exercício. Este artigo propõe-se a discutir alguns desses tópicos.

\section{EFEITOS DA ATIVIDADE FÍSICA NO INDIVÍDUO SEM DIABETES}

Inicialmente é interessante se definir alguns termos que aparecerão neste artigo. Atividade física é considerada como um movimento corporal não planejado, ao passo que o exercício corresponde ao movimento corporal estruturado e planejado. Exercício aeróbico consiste em um movimento rítmico, repetido e contínuo de diversos grupos musculares por, pelo menos, dez minutos, por exemplo, fazer uma caminhada, andar de bicicleta, nadar. Já o exercício resistido consiste em atividades que usam força muscular para mover um peso ou uma força contrária, por exemplo, musculação (2).

No início do exercício, a energia provém da glicose estocada na forma de glicogênio no músculo e no fígado. Quando o exercício se prolonga, os estoques de glicogênio se esgotam e a gordura pode passar a ser a principal fonte de energia (3).

Durante o exercício, a quantidade de insulina deve ser suficiente para permitir a entrada de glicose dentro da célula muscular, mas deve ser reduzida para liberar os estoques de glicogênio do fígado.

A produção de glucagon e adrenalina devem aumentar, pois esses dois hormônios permitem a libera- ção dos estoques de glicogênio. Assim, o aumento do consumo muscular de glicose é compensado pelo aumento equivalente da produção de glicose pelo fígado, desde o início do exercício, e a glicemia permanece estável (3). Esta regulação é perturbada no indivíduo com diabetes.

\section{EXERCÍ́CIO NO INDIVÍDUO COM DM1}

Durante a atividade física, os estoques de glicogênio são bloqueados pela presença da insulina e os músculos consomem glicose, por isso é eminente o risco de hipoglicemia. Isso ocorre se a dose da insulina ou o consumo de carboidrato não for alterado. A ingestão adicional de carboidrato, se a glicemia estiver $<100 \mathrm{mg} / \mathrm{dL}$, antes do exercício é fundamental $(2,4,5)$.

Algumas situações tornam a realização do exercício físico requerente de maior atenção. De acordo com recomendações anteriores da Associação Americana de Diabetes (ADA), indivíduos com glicemia maior que $250 \mathrm{mg} / \mathrm{dL}$ com presença confirmada de cetose devem evitar a realização do exercício físico e como precaução, glicemia > $300 \mathrm{mg} / \mathrm{dL}$ mesmo sem confirmação de cetose o exercício deve ser realizado com bastante cautela ou de preferência evitado $(4,6)$. As recomendações atuais da ADA enfocam não o nível glicêmico, mas sim a presença de cetose e a ausência do uso de insulina por 12 a 48 horas. Nesse caso, os exercícios, principalmente os vigorosos, podem piorar a hiperglicemia e a cetose. Entretanto, desde que o paciente esteja clinicamente bem, com diurese normal e com cetonas plasmáticas negativas não é necessário adiar o exercício fundamentado apenas na hiperglicemia (2).

Alguns fatores influenciam a resposta glicêmica ao exercício, como controle metabólico, insulina na forma de bólus anterior ao exercício, tipo e/ou intensidade do exercício, horário do exercício e fase do ciclo menstrual em mulheres $(7,8)$. 


\section{EFEITO DO EXERCÍ́CIO NO CONTROLE GLICÊMICO}

Resultados contraditórios têm sido relatados sobre os benefícios da atividade física no controle metabólico dos pacientes com DMl. Mosher e cols. (9) demonstraram efeitos benéficos na hemoglobina glicada (Alc) de 11 pacientes com DMl após 12 semanas de exercício aeróbico associado ao exercício resistido. Resultados benéficos similares foram demonstrados por Campaigne e cols. (10) em adolescentes com DMl após 12 semanas de jogos e atividades recreativas.

Avaliando crianças e adolescentes, Valerio e cols. (11) verificaram que a atividade física regular, por meio da participação em atividades esportivas, estava associada à melhora no controle metabólico, avaliado pela $\mathrm{Alc}$.

Em contraste, Laaksonen e cols. (12) não observaram redução na $\mathrm{Alc}$ após 12 semanas de um programa de treinamento em 20 pacientes com DMl, comparada com grupo controle. Melhora no consumo máximo de oxigênio $\left(\mathrm{VO}_{2} \mathrm{max}\right)$ sem melhora associada no controle glicêmico foi relatado por Rowland (13) e Zinman (14) após 12 semanas de exercício aeróbico, bicicleta e esteira, respectivamente. Concordante com outros autores, Ramalho e cols. (15) observaram que a atividade física, tanto no grupo submetido a treinamento aeróbico quanto no grupo em treinamento resistido, durante 12 semanas, não está associada à melhora da Alc. A ausência de melhora na Alc, em alguns estudos, pode ser explicada pela redução inadequada das doses de insulina para evitar hipoglicemia relacionada ao exercício, já que não existem protocolos bem estabelecidos para redução da dose de insulina.

Ligtenberg e cols. (16) demonstraram, com programa de exercícios, redução significante na Alc em indivíduos com diabetes melito tipo 2 (DM2), mas apenas após um ano de treinamento e não com seis meses. Isso sugere que pode ser necessário um período mais prolongado de atividade física regular antes do efeito no controle metabólico ser observado. Como a maioria dos estudos do efeito da atividade física em DMl tem duração inferior a seis meses, isso pode representar um período curto para percepção do efeito do exercício, embora outros estudos tenham verificado efeito benéfico, mesmo em períodos mais curtos (17).

\section{EFEITO DO EXERCíCIO NO PERFIL LIPÍDICO}

O efeito do exercício no perfil lipídico em DMl é controverso. Mosher e cols. (9) mostraram redução LDLc em adolescentes com DMl em programa de exercício aeróbico e resistido. Já outros autores não encontraram alteração no perfil lipídico após dez semanas de treinamento, tanto aeróbico quanto resistido, ou em uma combinação dos dois tipos de exercício em adolescentes (19). Neste estudo, os níveis de lipídios pré-tratamento não estavam alterados. Fripp e Hodgson (20) evidenciaram melhora significativa no perfil lipídico pós-programa de exercício, quando estudaram indivíduos com níveis de lipídios mais elevados antes do programa de exercício. Ramalho e cols. (15), em estudo prospectivo, avaliaram o efeito de 12 semanas de exercício aeróbico e resistido no controle metabólico e não observaram alteração no perfil lipídico.

Nesses estudos, a ausência de efeito dos exercícios no perfil lipídico pode estar relacionada à curta duração destes para que se possa observar este efeito ou a um perfil lipídico não alterado antes do programa de exercício. Talvez o maior efeito do exercício seja em indivíduos já com um perfil lipídico alterado antes do programa de exercícios e não naqueles cujo perfil lipídico seja normal. Além disso, como evidenciaram Laaksonen e cols. (12), a avaliação de outros parâmetros, como níveis de LDL-a ou ApoB, pode estar alterada.

\section{AVALIAÇÃo PRÉ-EXERCÍCIO}

A avaliação pré-exercício não é obrigatória, a não ser em pacientes que iniciem um programa de exercício de moderada a alta intensidade e possuam risco elevado para desenvolvimento de doença cardiovascular (DCV). Com base nesses critérios: idade maior que 35 anos ou idade maior que 25 anos e DMl há mais de 15 anos, presença de algum fator de risco para doença arterial coronariana, presença de retinopatia proliferativa ou nefropatia com microalbuminúria, doença arterial periférica e presença de neuropatia autonômica. A idade do paciente e o nível de atividade física prévia devem ser considerados $(2,4)$.

\section{AVALIAÇÃO DAS COMPLICAÇÕES CRÔNICAS PARA O EXERCíCIO}

A recomendação de teste de esforço de rotina é controversa. Alguns autores consideram que o teste de esforço não deveria ser recomendado de rotina para detectar isquemia em indivíduos assintomáticos, com baixo risco de DCV $(<10 \%$ de risco de eventos cardiovasculares em 10 anos), pois o risco de testes subseqüentes invasi- 
vos, em virtude dos resultados falso-positivos, pode compensar as expectativas benéficas para a detecção prévia de isquemia $(21,22)$.

Diminuição da sensibilidade dolorosa em extremidades resulta o risco de lesões de pele, infecções e artropatia de Charcot. Por isso, na presença de neuropatia periférica, devem ser encorajados os exercícios que não envolvam sobrecarga de peso em membros inferiores, como natação, andar de bicicleta ou exercícios de membros superiores (2). A presença de pulsos pedioso e tibial posterior não exclui alterações isquêmicas nos pés (5).

Exercícios aeróbicos vigorosos ou de resistência devem ser evitados na presença de retinopatia diabética proliferativa ou retinopatia grave não-proliferativa, por causa do risco de hemorragia vítrea ou do descolamento de retina (23). O grau de retinopatia tem sido usado para estratificar o risco da atividade física para uma prescrição individualizada (5).

A presença de neuropatia autonômica pode limitar a capacidade do indivíduo ao exercício e aumentar o risco de eventos cardiovasculares adversos durante o exercício. Neuropatia cardiovascular autonômica pode ser indicada pela presença de taquicardia de repouso (> $100 \mathrm{bpm}$ ), hipotensão ortostática (queda da pressão sistólica $>20 \mathrm{mmHg}$ com a ortostase) ou outros distúrbios do sistema nervoso autonômico (24).

Em pacientes com neuropatia autonômica, hipotensão e hipertensão depois de exercícios vigorosos são mais fáceis de ocorrer, especialmente quando iniciam um programa de exercício. Em virtude desses indivíduos terem problemas com a termorregulação, eles deveriam ser advertidos de que exercícios em temperaturas extremas devem ser evitados e que devem manter uma hidratação adequada (24).

\section{ADAPTAÇÃO DO TRATAMENTO A̧O EXERCÍCIO}

\section{Insulina}

Na maioria dos casos, o exercício dura de 30 minutos a 1 hora. Para adaptar bem a dose de insulina correspondente a este período, as de ação ultra-rápida (aspart ou lispro) são preferíveis. $\mathrm{O}$ esquema de tratamento mais favorável são múltiplas doses de injeção ultra-rápida associada à insulina basal, de preferência glargina, detemir ou bomba de insulina (25-27). As adaptações, no entanto, são possíveis com todas as insulinas, porém, quanto mais insulina basal o indivíduo usa, mais as adaptações são aleatórias.

\section{Adaptação da dose de insulina}

Apesar de o exercício ser considerado parte do tratamento em DMl, a ADA (28) enfatiza a necessidade do desenvolvimento de estratégias a fim de permitir aos indivíduos com DMl participarem com segurança em programas de atividade física, com redução do risco de hipoglicemia. $\mathrm{O}$ exercício aumenta o risco de hipoglicemia tanto durante quanto até 31 horas, no período de recuperação (29). Existem escassos dados na literatura com orientação para ajustes de insulina durante o exercício. Rabasa-Lhoret e cols. (30) propuseram, em seu estudo, um esquema para adaptação de doses de insulina relacionado à duração e à intensidade do exercício, quando realizado no período pós-prandial (Tabela 1 ).

Tabela 1. Sugestão para redução de pré-refeição da dose de insulina lispro em relação à intensidade e à duração do exercício pós-prandial.

\begin{tabular}{|c|c|c|}
\hline \multirow{2}{*}{$\begin{array}{c}\text { Intensidade do } \\
\text { exercício } \\
\left(\% \mathrm{VO}_{2 \text { max }}\right)\end{array}$} & \multicolumn{2}{|c|}{ \% redução da dose } \\
\hline & $\begin{array}{l}30 \text { minutos } \\
\text { de exercício }\end{array}$ & $\begin{array}{l}60 \text { minutos } \\
\text { de exercício }\end{array}$ \\
\hline 25 & $25^{\star}$ & 50 \\
\hline 50 & 50 & 75 \\
\hline 75 & 75 & \\
\hline
\end{tabular}

Fonte: Rabasa-Lhoret, 2001 (30).

Com base nas recomendações gerais de Wasserman e Abunrad (31) e de Zinman (14), a ADA (28) definiu que as adaptações ao exercício fossem fundamentadas na monitorização da glicemia capilar com objetivo de evitar hipoglicemia, sem estabelecer protocolo de redução de insulina bem definido.

A adaptação da dose de insulina ao exercício deve levar em consideração a sua intensidade, conforme proposto por Rabasa-Lhoret e cols. (30). Para entender como avaliar a intensidade do exercício verifique a Tabela 2.

Exercícios de alta intensidade podem aumentar a glicemia durante o exercício, não necessitando algumas vezes de redução da dose de insulina que cobre o período do exercício, ou seja, a insulina ultra-rápida ou rápida da refeição anterior ao exercício (29). 
Tabela 2. Classificação da intensidade da atividade física, com base nas atividades que durem até 60 minutos.

\begin{tabular}{|c|c|c|c|}
\hline \multicolumn{4}{|c|}{ Intensidade relativa } \\
\hline Intensidade & $\mathrm{VO}_{2 \text { máx }}(\%)^{\#}$ & Freqüência cardíaca $_{\text {máx }}(\%)^{*}$ & RPE ** \\
\hline Muito leve & $<20$ & $<35$ & $<10$ \\
\hline Leve & $20-39$ & $35-54$ & $10-11$ \\
\hline Moderado & $40-59$ & $55-69$ & $12-13$ \\
\hline Intenso & $60-84$ & $70-89$ & $14-16$ \\
\hline Muito intenso & $>85$ & $>90$ & $17-19$ \\
\hline Máximo*** & 100 & 100 & 20 \\
\hline
\end{tabular}

" $\mathrm{VO}_{2 \text { máx }}=$ consumo máximo de $\mathrm{O}_{2}$; ${ }^{*}$ Freqüência cardíaca máxima $\left(\mathrm{FC}_{\text {máx }}\right)=220$ - idade (Nota: É preferivel e recomendado que a $\mathrm{FC}$ máx $\mathrm{seja}$ medida durante um teste de alta intensidade); **Avaliação de Borg percebida é relativa à extensão da escala 6-20 (RPE); *** Média dos valores máximos conseguidos durante o exercício intenso por adultos saudáveis.

Fonte: ADA, 2002 (4).

Por outro lado, uma combinação de exercício de moderada e alta intensidades, conhecido como exercício intermitente, tem recebido pouca atenção de pesquisas. Essa característica dos exercícios correspondem a esportes de times ou brincadeiras espontâneas de crianças, merecendo, portanto, mais estudos.

É importante conhecer o efeito de cada tipo de exercício na glicemia para a realização da adaptação da insulina necessária. Guelfi e cols. (29) sugerem menor redução na glicemia com exercícios intermitentes de alta intensidade comparados a exercícios contínuos de moderada intensidade, e atribui esta diferença ao incremento na produção de glicose e redução na sua utilização no caso de exercícios intermitentes de alta intensidade.

Para saber qual a melhor adaptação é necessário realizar monitorização da glicemia antes, durante e após o exercício (28). A partir desses registros é possível perceber qual o melhor esquema para cada exercício. Caso haja variação na intensidade e/ou na duração, o esquema de insulina será outro e os registros devem ser feitos para o exercício com novas características. Cada exercício deve ser registrado em mais de uma oportunidade para que se observe a tendência de resposta glicêmica.

Dessa forma, deve-se estar bastante atento para a adaptação da dose de insulina na prática de exercício, pois os diversos fatores ligados ao exercício devem ser considerados, quais sejam, intensidade, duração, tipo e horário. Nenhum protocolo proposto fundamentado em apenas um parâmetro poderá se adaptar a todos os tipos de exercício.

\section{REPOSIÇÃO DE CARBOIDRATO PARA O EXERCÍ́CIO}

Para o exercício planejado, a redução na dose de insulina é o método mais adequado para a redução da hipoglicemia. No entanto, para o exercício não programado a ingestão adicional de carboidrato $(\mathrm{CHO})$ é necessária. Segundo recomendações da ADA (2) para um exercício de moderada intensidade, a ingestão adicional de 2 a $3 \mathrm{mg} / \mathrm{kg} / \mathrm{min}$ de CHO é suficiente. Ou seja, uma pessoa de $70 \mathrm{~kg}$ precisaria de 10 a $15 \mathrm{~g}$ de carboidrato por hora de exercício moderado. Para exercícios de atividade intensa maior quantidade de $\mathrm{CHO}$ pode ser necessária (2).

Se os níveis de glicose sanguínea estiverem $<100$ $\mathrm{mg} / \mathrm{dl}$ durante o exercício, $15 \mathrm{~g}$ de $\mathrm{CHO}$ devem ser administrados, como um açúcar de absorção rápida. Para crianças menores, essa quantidade pode ser menor. Em torno de $10 \mathrm{~g}$ de $\mathrm{CHO}$. Para atividade vigorosa com duração maior de 30 minutos, um adicional de $15 \mathrm{~g}$ de $\mathrm{CHO}$ pode ser necessário (32). 
O uso de uma fonte de absorção rápida de hidrato de carbono, como uma bebida isotônica, pode ser muito útil em prevenir a hipoglicemia durante e após o exercício (32).

No entanto, recomendações específicas, considerando a quantidade de suplementação com $\mathrm{CHO}$ durante o exercício, variam entre pesquisadores $(33,34)$. Wasserman e Abunrad (31) sugeriram que a reposição pode ser calculada sobre o uso relativo de $\mathrm{CHO}$ para o suprimento energético de cada atividade física. Por exemplo, em um exercício de intensidade moderada, em torno de $50 \%$ do dispêndio energético ocorre por oxidação de carboidratos. Dessa forma, para o dispêndio calórico de $12 \mathrm{kcal} / \mathrm{min}^{-1}$, em que $50 \%$ desse provenha do $\mathrm{CHO}$, significa dizer que a cada minuto, nessa atividade, $6 \mathrm{kcal}$ proveniente de $\mathrm{CHO}$ serão necessárias. Como cada grama de $\mathrm{CHO}$ possui $4 \mathrm{kcal}$, a necessidade de $\mathrm{CHO}$ em gramas para cada minuto dessa atividade específica seria de $1,5 \mathrm{~g}$, ou seja, a cada 30 minutos uma reposição de 45 g seria necessária (35).

Young (34) sugere o consumo de um lanche contendo 40 a $69 \mathrm{~g}$ de $\mathrm{CHO}$ antes de um exercício de intensidade moderada, enquanto Horton (33) propõe a ingestão de 35 a $40 \mathrm{~g}$ de $\mathrm{CHO}$ a cada 30 minutos. Wallberg-Henriksson e cols. (17) sugerem a diminuição da dose de insulina e o consumo suplementar de CHO para exercício moderado de 30 minutos para duas horas de ciclismo.

Kemmer e Berger (36) propõem um ajuste na dose de insulina e no consumo de $\mathrm{CHO}$ de 15 a 30 g a cada 30 minutos para exercícios prolongados. Andrade e cols. (35) examinaram a glicemia em adolescentes com DMl que ingeriram bebidas esportivas com $6 \%$ de $\mathrm{CHO}$ durante e após o exercício. A quantidade média de $\mathrm{CHO}$ ingerida foi de $39,56 \pm 8,08 \mathrm{~g}$ durante os primeiros 45 minutos de exercício. Eles concluíram que a ingestão de uma bebida contendo $6 \%$ de $\mathrm{CHO}$ atenuou a redução da glicemia induzida pelo exercício em adolescentes com DMl. Uma vantagem da suplementação de $\mathrm{CHO}$ sob a forma de fluidos é a manutenção da hidratação (35).

Quando o atleta já está em treinamento, a maioria dos ajustes para impedir a hipoglicemia induzida pelo exercício será feita pelo suplemento dietético com hidrato de carbono. Isso envolverá uma quantidade especificada de hidrato de carbono em quantidade apropriada a prevenir a redução da glicemia.
A quantidade de hidrato de carbono requerida gira em torno de 15 a $60 \mathrm{~g}$. As bebidas que contêm o hidrato de carbono $5 \%$ a $10 \%$ podem ser boas escolhas, pois ajudam nas perdas fluidas durante o exercício. A maioria das bebidas comerciais desportivas tem o hidrato de carbono a aproximadamente $6 \%$ a $7 \%$. As quantidades são identificadas facilmente nos rótulos. As bebidas que contêm o hidrato de carbono de $10 \%$ podem causar desconforto gastrintestinal e devem ser diluídas (6).

Uma hidratação apropriada é essencial e pode ser feita com $500 \mathrm{ml}$ de líquido, duas horas antes do exercício. Durante o exercício, o líquido deve ser reposto freqüentemente em uma quantidade suficiente para compensar perdas no suor (5).

\section{AUTOMONITORIZAÇÃO}

Dos episódios de hipoglicemia na população pediátrica, $10 \%$ a $20 \%$ são associados a exercícios, os quais são geralmente maiores que os de intensidade, duração e freqüência usuais (32). De outro lado, a ocorrência de hiperglicemia ligada ao exercício deve ser checada imediatamente nos casos de exercícios vigorosos. Neste caso pode ocorrer hipoglicemia dentro de uma a seis horas após o exercício por causa da depleção hepática do glicogênio. Estas diferentes respostas ao exercício requerem freqüente ajuste na dose da insulina, fazendo-se necessário o uso da monitorização da glicemia capilar (32).

A monitorização antes e após o exercício é fundamental para identificar quando será necessária a mudança na dose de insulina ou a ingestão de alimentos, bem como para identificar a resposta glicêmica a diferentes condições de exercícios (4).

A resposta glicêmica ao exercício é afetada por diversos fatores, como a intensidade, a duração, o horário do exercício, as condições ambientais, o estresse e a absorção da insulina e de suplementos dietéticos. Para observar estas diferentes respostas é necessário uma monitorização glicêmica.

\section{BOMBA E EXERCÍ́CIO}

A bomba de insulina permite maior liberdade e comodidade quanto ao conteúdo das refeições e à prática de exercícios físicos, visto que esta última é favorecida também em virtude dessa terapêutica utilizar-se apenas de um sítio de aplicação (37). 
Durante a realização de atividades esportivas, o tempo a ser mantido sem infusão de insulina e a ingestão ou não de alimentos está na dependência do tipo e da duração da atividade a ser desenvolvida, bem como da idade e do sexo do paciente $(38,39)$. Em exercícios de maior contato físico com risco de dano à bomba, como lutas e futebol, pode ser indicada sua retirada. A natação e a hidroginástica também exigem sua retirada, exceto nos casos em que utilizam a bomba à prova d'água. Outra possibilidade, além da suspensão da infusão de insulina da bomba, é a utilização de um basal temporário, no qual se programa uma taxa de infusão menor do que a utilizada para aquele horário (40). É importante ressaltar que o paciente não deve permanecer desconectado da bomba por tempo prolongado pelo risco de hiperglicemia posterior (39). Admon e cols. (40) não verificaram vantagem no uso de um basal temporário com $50 \%$ da taxa basal em relação à suspensão do basal. Os autores chamam a atenção para a possibilidade de hipoglicemia tardia em exercício prolongado, necessitando, portanto, de monitorização glicêmica tardia e provável redução do basal tardio ao exercício (40).

\section{ESCOLHA DO TIPO DE EXERCÍCIO}

Outra questão que permanece não resolvida no tema "exercício e diabetes" está relacionada ao exercício mais benéfico para o controle metabólico: exercício aeróbico ou resistido. Estudos que avaliam o efeito do exercício no controle metabólico em pacientes com DMl usaram treinamento aeróbico associado a treinamento resistido $(9,10,12,13)$. São escassos na literatura dados sobre o efeito de exercício resistido, sem exercício aeróbico, no controle metabólico em DMl. Tendo em vista que DMl é mais prevalente em pessoas jovens que freqüentam academias, a resposta sobre o efeito do treinamento resistido no controle metabólico em DMl torna-se muito importante. Ramalho e cols. (15) avaliaram o efeito do treinamento resistido, isolado, sem treinamento aeróbico, no controle metabólico em pacientes com DMl e não observaram alteração significativa na Alc após o treinamento, embora a tendência à redução da Alc tenha sido observada no grupo em treinamento resistido $(8,2 \% \pm 2,9 \%$ pré-treinamento para $7,6 \pm 1,6 \%$ pós-programa de treinamento). Como este treinamento teve duração de 12 semanas, talvez, com um tempo mais prolongado, o efeito benéfico na Alc ficasse evidente.

\section{DIABETES E ESPORTES DESACONSELHADOS}

Todos os tipos de atividade física, incluindo atividades de lazer, esportes de recreação, performance de competição profissional, podem ser executados por pessoas com DMl, pois os benefícios dos exercícios atuam nos fatores de risco para doença macrovascular, dislipoproteinemia, hipertensão, obesidade e melhora da performance cardíaca. Além disso, esses aspectos promovem melhora na qualidade de vida e contribuem para a disciplina dos jovens (39). Deve-se, no entanto, atentar para a presença de complicações, como já discutido anteriormente (4).

Mesmo com a possibilidade de realização de todos os esportes pelo indivíduo com diabetes, alguns são pouco estimulados por diversos fatores, como: 1 . Por provocar redução de atenção, a hipoglicemia pode colocar em risco a segurança nos esportes que envolvem pilotar carro, motocicleta, avião. 2. Pela dificuldade de correção da hipoglicemia em esportes, como mergulho, pára-quedismo, escaladas. No alpinismo, acima de $2.500 \mathrm{~m}$, os sintomas de hipoglicemia podem não ser tão evidentes; neste caso pensar em hipoglicemia diante de fadiga, dor de cabeça, vertigem ou irritabilidade. Salientar que acima de $1.500 \mathrm{~m}$ os glicosímetros perdem em precisão. Para a prática desses esportes é necessário um excelente autoconhecimento por meio da automonitorização.

\section{REFERÊNCIAS}

1. Moy CS, Songer TJ, La Porte R, Dorman JS, Kriska AM, Orchard TJ, et al. Insulin-dependent diabetes mellitus, physical activity and death, Am. J. Epidemiol. 1993;137:74-81.

2. American Diabetes Association: standards of medical care in diabetes (position statement). Diabetes Care. 2007;30 Suppl 1:S4-41.

3. Robergs RA, Robert SO. Respostas sistêmicas ao exercício. Princípios fundamentais de fisiologia do exercício. In: Robergs RA e Robert SO, editores. São Paulo: Phorte; 2002. p. 34-46.

4. American Diabetes Association: diabetes mellitus and exercise (position statement). Diabetes Care. 2002;25 Suppl 1:S64-8.

5. American Diabetes Association: physical activity/exercise and diabetes (position statement). Diabetes Care. 2004;27 Suppl 1:S58-62.

6. Hornsby WGJ, Chetlin RD. Management of competitive athletes with diabetes. Diabetes Spectr. 2005;18:102-7.

7. Moberg E, Kollind M, Lins PE, Adamson U. Day-to-day variation of insulin sensitivity in patients with type 1 diabetes: role of gender and menstrual cycle. Diabetic Med. 1995;12:224-8. 
8. Widom B, Diamond MP, Simonson DC. Alterations in glucose metabolism during menstrual cycle in women with IDDM. Diabetes Care. 1992;15:213-20.

9. Mosher PE, Nash MS, Perry AC, La Pierriere AR, Goldberg BB. Aerobic circuit training: effect on adolescents with well-controlled insulin-dependent diabetes mellitus. Arch. Phys Med Rehabil. 1998;79:652-7.

10. Campaigne BN, Gilliam TB, Spencer ML, Lampman RM, Stork MA. Effects of a physical activity program on metabolic control and cardiovascular fitness in children with insulin-dependent diabetes mellitus. Diabetes Care. 1984;7:57-62.

11. Valerio G, Spagnuolo MI, Lombardi F, Spadaro R, Siano M. Physical activity and sports participation in children and adolescents with type 1 diabetes mellitus. Nutr Metab Cardiovasc Dis. 2007;17:376-82.

12. Laaksonen DE, Attala M, Insane LK, Mudstone J, Sen CK, Lake TA, et al. Aerobic exercise and the lipid profile in type-1 diabetic men: a randomized controlled trial. Med Sci Sports Exerc. 2000;32:1541-8.

13. Rowland T. Physical fitness in children: implications for the prevention of coronary artery disease. Curr Prob Pediatr. 1981;11:1-5.

14. Zinman B, Zuniga-Guajardo S, Kelly D. Comparison of the acute and long-term effects of exercise on glucose control in type-1 diabetes. Diabetes Care. 1984;7:515-9.

15. Ramalho AC, De Lourdes Lima M, Nunes F, Cambuí Z, Barbosa $C$, Andrade $A$, et al. The effect of resistance versus aerobic training on metabolic control in patients with type-1 diabetes mellitus. Diabetes Res Clin Pract. 2006;72:271-6.

16. Ligtenberg PC, Blans M, Hoekstra JB, van der Tweel I, Erkelens DW. No effect of long term physical activity on the glycemic control in type-1 diabetes patients a cross-sectional study, Neth J Med.1999;2:59-63.

17. Wallberg-Henriksson $H$, Gurnasson R, Rossner S, Wahren J. Long term physical training in female type-1 (insulin-dependent) diabetic patients: absence of significant effect on glycemic control and lipoprotein levels. Diabetologia. 1986;7:515-9.

18. Wallberg-Henriksson $\mathrm{H}$. Acute exercise: fuel homeostasis and glucose transport in insulin-dependent diabetes mellitus. Med Sci Sports Exerc. 1989;21:356-61.

19. Lee A, Craig BW, Lucas J, Pohlman R, Stelling H. The effect of endurance training and weight training upon the blood lipid profiles of young male subjects. J Appl Sport Sci Res.1990;4: 68-75.

20. Fripp RP, Hodgson JL. Effects of resistance training on plasma lipid and lipoprotein levels in male adolescent. J Pediatr. 1987;111:926-31.

21. US Preventive Services Task Force: screening for coronary heart disease: recommendation statement. Ann Intern Med. 2004;140:569-72.

22. Fowler-Brown A, Pignone M, Pletcher M, Tice JA, Sutton SF, Lohr KN. Exercise tolerance testing to screen for coronary heart disease: a systematic review for the technical support for the U.S. Preventive Services Task Force. Ann Intern Med. 2004;140:W9-24.

23. Aiello LP, Wong J, Cavallerano J, Bursell SE, Aiello LM. Retinopathy. In: Ruderman N, Devlin JT, Schneider SH, Kriska A, editores. Handbook of exercise in diabetes. $2^{\mathrm{a}}$ ed. Alexandria, VA: American Diabetes Association; 2002. p. 401-13.

24. Boulton AJ, Vinik Al, Arezzo JC, Bril V, Feldman EL, Freeman $R$, et al. Diabetic neuropathies: a statement by the American Diabetes Association. Diabetes Care. 2005;28:956-62.
25. Wilde MI, McTavish D. Insulin lispro: a review of its pharmacological properties and therapeutic use in the management of diabetes mellitus. Drugs. 1997;54:597-614.

26. Jacobs MAJM, Keulen ETP, Kanc K, Casteleijn S, Scheffer $P$, Deville W, et al. Metabolic efficacy of preprandial administration of Lys(B28), Pro(B29) human insulin analog in IDDM patients. Diabetes Care. 1997;20:1279-86.

27. Brunelle RL, Llewelyn J, Anderson JH, Gale EAM, Koivisto VA. Meta-analysis of the effect of insulin lispro on severe hypoglycemia in patients with type 1 diabetes. Diabetes Care. 1998;21:1726-31.

28. American Diabetes Association: standards of medical care in diabetes. Diabetes Care. 2005;28:S4-36.

29. Guelfi KJ, Ratnam N, Smythe GA, Jones TW, Fournier PA. Effect of intermittent high-intensity compared with continuous moderate exercise on glucose production and utilization in individuals with type 1 diabetes. Am J Physiol Endocrinol Metab. 2007;292:E865-70.

30. abasa-Lhoret R, Ducros F, Bourque J, Chiasson JL. Guidelines for premeal insulin dose reduction for postprandial exercise of different intensities and durations in type 1 diabetic subjects treated intensively with a basal-bolus insulin regimen (ultralente-lispro). Diabetes Care. 2001;24:625-30.

31. Wasserman DH, Abunrad NN. Physiological bases for the treatment of the physically active individuals with diabetes. Sports Med. 1989;7:376-92.

32. Silverstein J, Klingensmith G, Copeland KC, Plotnick L, Kaufman $F$, Laffel L, et al. American Diabetes Association: care of children and adolescents with type 1 diabetes mellitus: a statement of the American Diabetes Association. Diabetes Care. 2005;28:186-212.

33. Horton ES. Role and management of exercise in diabetes mellitus. Diabetes Care. 1988;11:201-11.

34. Young JC. Exercise prescription for individuals with metabolic disorders. Sports Med. 1995;19:43-54.

35. Andrade R, Laitano O, Meyer F. Efeito da hidratação com carboidratos na resposta glicêmica de diabéticos tipo 1 durante $o$ exercício. Rev Bras Med Esporte. 2005;11(1):61-5.

36. Kemmer FW, Berger M. Exercise and diabetes mellitus: physical activity as a part of daily life and its role in the treatment of diabetic patients. Int J Sports Med. 1983;4:77-88.

37. Renner R, Pfuntzner A, Trautmann M, et al. Use of insulin lispro in continuous subcutaneous insulin infusion treatment. Results of a multicenter trial. Diabetes Care. 1999;22:784-8.

38. Liberatore Júnior RDR, Damiani D. Bomba de infusão de insulina em diabetes melito tipo 1. JPED. 2006;82:249-54.

39. Tsalikian E, Kollman C, Tamborlane WB, Beck RW, FialloScharer R, Fox L, et al. Prevention of hypoglycemia during exercise in children with type 1 diabetes by suspending basal insulin. Diabetes Care. 2006;29:2200-4.

40. Admon $G$, Weinstein $Y$, Falk B, Weintrob N, Benzaquen $H$, Ofan $\mathrm{R}$, et al. Exercise with and without an insulin pump among children and adolescents with type 1 diabetes mellitus. Pediatrics. 2005;116:348-55.

Endereço para correspondência:

Ana Claudia R. Ramalho

Rua Piratancara, 112

40295-040 - Horto Florestal, Salvador, BA

E-mail: acrramalho@superig.com.br 